\title{
How to stay out of trouble in RIXS calculations within the equation-of-motion coupled-cluster damped response theory framework? Safe hitchhiking in the excitation manifold by means of core-valence separation
}

Kaushik D. Nanda ${ }^{a}$, Marta L. Vidal ${ }^{b}$, Rasmus Faber $^{b}$, Sonia Coriani ${ }^{b}$, and Anna I. Krylova,c

${ }^{a}$ Department of Chemistry, University of Southern California, Los Angeles, California 90089-0482

${ }^{b}$ DTU Chemistry - Department of Chemistry, Technical

University of Denmark, DK-2800, Kongens Lyngby, Denmark

${ }^{c}$ The Hamburg Centre for Ultrafast Imaging, Luruper Chaussee 149, 22671 Hamburg, Germany

We present a novel approach for computing resonant inelastic X-ray scattering (RIXS) cross sections within the equation-of-motion coupled-cluster (EOM-CC) framework. The approach is based on recasting the sum-over-state expressions for RIXS moments into a compact form by using damped response theory. Damped response formalism allows one to circumvent problems of divergent behavior of the response equation in the resonant regime. However, the convergence of response equations in the X-ray frequency range is often erratic due to the resonant nature of the virtual core-excited states embedded in the valence ionization continuum. We demonstrate that this problematic behavior can be avoided by extending the core-valence separation (CVS) scheme, which decouples the valence-occupied and core-occupied excitation manifolds, into the response domain. The accuracy of the CVS-enabled damped response theory, implemented within the EOM-EE-CCSD (EOM-CC for excitation energies with single and double excitations) framework, is assessed by comparison against damped EOM-EE-CCSD response calculations. The capabilities of the new approach are illustrated by calculations of RIXS cross sections for benzene and benzene radical cation. 


\section{INTRODUCTION}

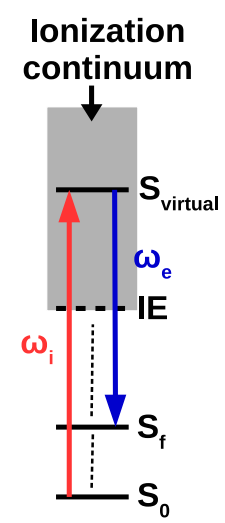

FIG. 1: RIXS is a non-linear process involving two photons: The excitation photon promotes the system from the ground state $\mathrm{S}_{0}$ into the virtual state $S_{\text {virtual }}$ and the system emits a photon by relaxing into an excited state $S_{f}$. The difference in the incident and emitted photon frequencies equals the excitation energy of final state $\left(\omega_{i}-\omega_{e}=E_{f}-E_{0}\right)$. The system can also relax back to the ground state giving rise to the REXS (resonant elastic X-ray scattering) signal. Whereas the final excited states lie below the ionization energy and are, therefore, bound with respect to electron ejection, the high-lying core-excited intermediate state $S_{\text {virtual }}$ is embedded inside the ionization continuum shown by the gray box.

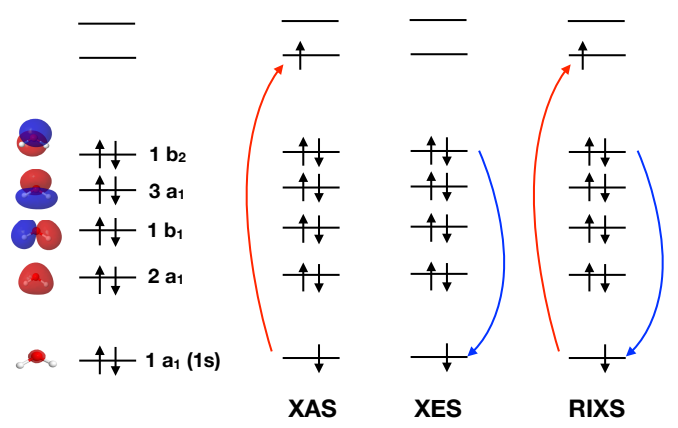

FIG. 2: Molecular orbital picture of the XAS, XES, and RIXS processes illustrated by using water. XAS entails excitation of core electrons into virtual molecular orbitals. The XES signal is produced by the relaxation of valence electrons into a core hole. RIXS process can be thought of as a two-step process coherently combining XAS and XES events.

Resonant inelastic X-ray scattering ${ }^{1-4}$ (RIXS) is a non-linear two-photon spectroscopy 
based on the transitions involving core-level states. As illustrated in Fig. 1, RIXS is a one-photon-in/one-photon-out process. The energy difference between the absorbed and emitted photons (i.e., energy loss spectrum) characterizes the differences between the valence electronic states. By virtue of using X-ray photons, RIXS exploits highly localized and element-specific nature of the core orbitals ${ }^{5}$. Although RIXS is a coherent scattering process, it can be thought of as an initial excitation of a core-level electron into a valence orbital followed by radiative decay of another valence electron to fill the core hole. This molecular orbital picture is shown in Fig. 2: the rightmost panel shows electronic configuration of the virtual state $S_{\text {virtual }}$ and the arrows illustrate absorption and emission transitions. In this respect, one can (crudely) describe RIXS as simultaneous union of the X-ray absorption and X-ray emission events. This connection between XAS (X-ray absorption spectroscopy), XES (X-ray emission spectroscopy), and RIXS is illustrated in Fig. 2 using molecular orbital diagram of water molecule. Thus, RIXS spectroscopy probes the unoccupied valence orbitals as in XAS, the occupied valence orbitals as in XES, and their correlation. Owing to its nonlinear nature, the selection rules in RIXS differ from those in linear spectroscopies. For example, RIXS can provide the information about the transitions between valence excited states that are optically dark in one-photon spectroscopies. The spatial localization of core orbitals and their sensitivity to the chemical environment endows RIXS with sensitivity to probe the local environment of an atom, its oxidation state, and the bonding pattern around it. Initial applications of RIXS were limited to studying charge-transfer and crystal-field transitions in metal oxides, however, advances in X-ray light sources and instrumentation have afforded studying ultrafast nuclear dynamics with RIXS.

The increasing popularity of X-ray spectroscopic tools ${ }^{6-9}$ such as RIXS, XAS, and XES have stimulated the development of quantum-chemical theory and electronic structure tools for modeling these spectra ${ }^{10,11}$ As in the case of VUV-based techniques ${ }^{12}$, theoretical modeling is required to unambiguously relate experimental measurements to molecular structures. However, as nicely described in a recent review ${ }^{10}$ of the theoretical modeling of X-ray spectroscopies, accurate description of core states is much more challenging than the description of valence states. Despite significant progress, the theoretical tools are lagging behind the experimental capabilities, creating a bottleneck for maximizing the scientific impact of multibillion advanced light sources facilities.

The challenges for electronic structure methods in modeling molecular processes in the X- 
ray regime include (1) the prevalence of open-shell electronic states, (2) the difficulty in computing high-lying core-excited states with standard solvers, (3) the strong orbital-relaxation effects due to strongly perturbed atomic core, (4) the strong sensitivity of the signal to environmental effects, and (5) the metastable character of the core-excited states embedded inside the valence ionization continuum ${ }^{13,14}$. Furthermore, computational methods for modeling multiphoton X-ray techniques such as RIXS face additional challenges such as (1) the increased complexity of formalisms and computer codes and (2) the non-convergence of response solutions due to the presence of the response states (or virtual states) in the valence ionization continuum. Whereas analytical implementations of the state-of-the-art algebraic diagrammatic construction ${ }^{15}$ (ADC) and coupled-cluster ${ }^{16}$ methods for computing RIXS cross sections for small to medium-sized systems within the damped response theory framework have been reported ${ }^{17,18}$, the issue of diverging response solutions has remained so far unaddressed.

The equation-of-motion coupled-cluster (EOM-CC) methods ${ }^{19-24}$ provide a robust singlereference multi-state strategy for computing multiconfigurational wave functions, excitation energies, electron affinities, and ionization potentials of a variety of closed- and open-shell systems. The EOM-CC framework not only gives reliable lower-order properties such as solvatochromic shifts ${ }^{25}$, dipole moments, and transition dipole moments ${ }^{20}$ but also higher-order properties $^{26}$ such as two-photon absorption cross sections ${ }^{27-32}$, spin-orbit couplings ${ }^{33-36}$, nonadiabatic couplings ${ }^{37-39}$, static and dynamical polarizabilities ${ }^{40-43}$. Whereas the bulk of prior developments and applications of the EOM-CC methods as well as of the closely related coupled-cluster response theory were in the VUV regime, these methods are now being extended to the X-ray regime and their performance is being explored for computing, for example, XAS ${ }^{14,44-49}, \mathrm{XES}^{16}$, and $\mathrm{RIXS}^{16}$ spectra.

Building on the EOM-CC damped response implementation reported by Faber and Coriani $^{16}$, here, we present a novel damped response implementation for computing RIXS cross sections with a variant of the EOM-CC singles and doubles method for electronic excitation $^{20-22,50,51}$ (EOM-EE-CCSD) that provides converged response solutions by judicious exploitation of the excitation manifold. Our strategy to get converged response solutions for RIXS calculations is to employ the core-valence separation (CVS) scheme originally proposed by Cederbaum et al. ${ }^{52}$ This approach has been employed to compute resolved core-excited and core-ionized states within the framework of EOM-CC theory ${ }^{14,48}$ but has 
not yet been employed for multiphoton X-ray processes such as RIXS. The CVS approximation projects out the valence ionization continuum and enables the resolution of core-excited (real or response) states. Specifically, we extend the recently reported CVS-EOM-EE-CCSD method with frozen-core (fc) approximation (fc-CVS-EOM-EE-CCSD) $)^{14}$ to the calculation of RIXS cross sections. This idea is also explored within the coupled-cluster singles and doubles complex-polarization propagator approach by Faber and Coriani ${ }^{53}$.

Here, we present the theory of RIXS cross sections within the EOM-EE-CCSD and fcCVS-EOM-EE-CCSD methods. We validate the implementation on a small test system $(\mathrm{LiH})$ and then compare the quality of EOM-EE-CCSD and fc-CVS-EOM-EE-CCSD RIXS spectra for the water molecule. The method is implemented in the Q-Chem electronic structure package ${ }^{54,55}$. Our production-level implementation is capable of treating both closed- and open-shell references. As an illustrative example, we compute and discuss RIXS spectra of benzene and its cation, highlighting the capabilities of the fc-CVS-EOM-EE-CCSD method.

\section{THEORY}

\section{A. CCSD and EOM-EE-CCSD theory}

The ground-state coupled-cluster wave function is given by

$$
\Psi_{0}=e^{\hat{T}}\left|\Phi_{0}\right\rangle
$$

where $\hat{T}$ is the cluster operator given in terms of creation $\left(a^{+}, b^{+}\right)$and annihilation $(i, j)$ operators as follows:

$$
\hat{T}=\hat{T}_{1}+\hat{T}_{2} ; \quad \hat{T}_{1}=\sum_{i a} t_{i}^{a} a^{+} i ; \quad \hat{T}_{2}=\frac{1}{4} \sum_{i j a b} t_{i j}^{a b} a^{+} b^{+} j i
$$

The $\hat{T}$ operator satisfies the following coupled-cluster equation:

$$
\left\langle\Phi_{\nu}|\bar{H}| \Phi_{0}\right\rangle=0,
$$

where $\nu$ spans the singles and doubles excitation manifold for CCSD and $\bar{H}=e^{-T} H e^{T}$ is the similarity-transformed Hamiltonian. 
In the EOM-CCSD approach for excitation energies, the target excited states are described as excitations from the CCSD wave function as follows:

$$
L^{k} \bar{H}=L^{k} E_{k}
$$

and

$$
\bar{H} R^{k}=E_{k} R^{k}
$$

where $L$ and $R$ are the EOM-CCSD left (de-excitation), and EOM-CCSD right (excitation) operators

$$
\hat{L}=l_{0}+\hat{L}_{1}+\hat{L}_{2} ; \quad \hat{L}_{1}=\sum_{i a} l_{i}^{a} i^{+} a ; \quad \hat{L}_{2}=\frac{1}{4} \sum_{i j a b} l_{i j}^{a b} i^{+} j^{+} b a
$$

and

$$
\hat{R}=r_{0}+\hat{R}_{1}+\hat{R}_{2} ; \quad \hat{R}_{1}=\sum_{i a} r_{i}^{a} a^{+} i ; \quad \hat{R}_{2}=\frac{1}{4} \sum_{i j a b} r_{i j}^{a b} a^{+} b^{+} j i
$$

Note that for the CCSD reference state $(k=0), \hat{R}=r_{0}$ and $\hat{L}=1+\hat{\Lambda}$, where $\hat{\Lambda}=\hat{\Lambda}_{1}+\hat{\Lambda}_{2}$ is the $\operatorname{CCSD} \Lambda$ operator. For EOM-CCSD states, $l_{0}=0$.

\section{B. RIXS within EOM-EE-CCSD damped response theory}

The RIXS cross section as a function of scattering angle $\theta$ (the angle between polarization of the incident and scattered photons) is given in terms of RIXS transition strengths $S_{a b, c d}^{i f}$ as follows:

$$
\sigma^{R I X S}(\theta)=\frac{1}{15} \frac{\omega_{e}}{\omega_{i}} \sum_{x y}\left[\left(2-\frac{1}{2} \sin (\theta)^{2}\right) S_{x y, x y}^{i f}+\left(\frac{3}{4} \sin (\theta)^{2}-\frac{1}{2}\right)\left(S_{x y, y x}^{i f}+S_{x x, y y}^{i f}\right)\right]
$$

where $\omega_{e}$ and $\omega_{i}$ are frequencies of emitted and incident photons and the indices $x, y$ denote the Cartesian components ${ }^{15,16}$. Within the non-Hermitian EOM-CC theory, $S_{a b, c d}^{i f}$ are given by

$$
S_{a b, c d}^{i f}=\frac{1}{2}\left(M_{a b}^{i \leftarrow f} M_{c d}^{f \leftarrow i}+\left(M_{c d}^{i \leftarrow f}\right)^{*}\left(M_{a b}^{f \leftarrow i}\right)^{*}\right)
$$

where $^{*}$ denotes complex conjugation ${ }^{56}$. Here, $M_{a b}^{f \leftarrow i}$ and $M_{a b}^{i \leftarrow f}$ are the right and left RIXS moments given by the sum-over-state expressions, which within the EOM-CC framework 
assume the following form

$$
\begin{array}{r}
M_{x y}^{f \leftarrow i}\left(\omega_{i, x}+i \gamma,-\omega_{e, y}-i \gamma\right) \\
=-\sum_{n \geq 0}\left(\frac{\left\langle\Phi_{0} L^{f} e^{-T}\left|\mu^{y}\right| e^{T} R^{n} \Phi_{0}\right\rangle\left\langle\Phi_{0} L^{n} e^{-T}\left|\mu^{x}\right| e^{T} R^{i} \Phi_{0}\right\rangle}{\Omega_{n i}-\omega_{i, x}-i \gamma}\right. \\
\left.\quad+\frac{\left\langle\Phi_{0} L^{f} e^{-T}\left|\mu^{x}\right| e^{T} R^{n} \Phi_{0}\right\rangle\left\langle\Phi_{0} L^{n} e^{-T}\left|\mu^{y}\right| e^{T} R^{i} \Phi_{0}\right\rangle}{\Omega_{n i}+\omega_{e, y}+i \gamma}\right)
\end{array}
$$

and

$$
\begin{aligned}
& M_{x y}^{i \leftarrow f}\left(-\omega_{i, x}-i \gamma, \omega_{e, y}+i \gamma\right)\left(\frac{\left\langle\Phi_{0} L^{i} e^{-T}\left|\mu_{x}\right| e^{T} R^{n} \Phi_{0}\right\rangle\left\langle\Phi_{0} L^{n} e^{-T}\left|\mu_{y}\right| e^{T} R^{f} \Phi_{0}\right\rangle}{\Omega_{n i}-\omega_{i, x}-i \gamma}\right. \\
&=-\sum_{n \geq 0} \\
&\left.\quad+\frac{\left\langle\Phi_{0} L^{i} e^{-T}\left|\mu_{y}\right| e^{T} R^{n} \Phi_{0}\right\rangle\left\langle\Phi_{0} L^{n} e^{-T}\left|\mu_{x}\right| e^{T} R^{f} \Phi_{0}\right\rangle}{\Omega_{n i}+\omega_{e, y}+i \gamma}\right) .
\end{aligned}
$$

Here, $\Omega_{n m}=E_{n}-E_{m}$ is the energy difference between state $n$ and $m$ and the photon frequencies ( $\omega$ 's) are augmented with the phenomenological damping term $i \gamma$.

Introducing the identity operator, $\mathbf{1}=\left|\Phi_{\rho}\right\rangle\left\langle\Phi_{\rho}\right|$, in Eqs. (10) and (11), we obtain

$$
\begin{aligned}
& M_{x y}^{f \leftarrow i}\left(\omega_{i, x}+i \gamma,-\omega_{e, y}-i \gamma\right) \\
& =-\sum_{\rho \nu}\left(\left\langle\Phi_{0} L^{f} e^{-T}\left|\mu^{y}\right| e^{T} \Phi_{\rho}\right\rangle \sum_{n \geq 0} \frac{\left\langle\Phi_{\rho} \mid R^{n} \Phi_{0}\right\rangle\left\langle\Phi_{0} L^{n} \mid \Phi_{\nu}\right\rangle}{\Omega_{n i}-\omega_{i, x}-i \gamma}\left\langle\Phi_{\nu} e^{-T}\left|\mu^{x}\right| e^{T} R^{i} \Phi_{0}\right\rangle\right. \\
& \left.\quad+\left\langle\Phi_{0} L^{f} e^{-T}\left|\mu^{y}\right| e^{T} \Phi_{\rho}\right\rangle \sum_{n \geq 0} \frac{\left\langle\Phi_{\rho} \mid R^{n} \Phi_{0}\right\rangle\left\langle\Phi_{0} L^{n} \mid \Phi_{\nu}\right\rangle}{\Omega_{n i}+\omega_{e, y}+i \gamma}\left\langle\Phi_{\nu} e^{-T}\left|\mu^{x}\right| e^{T} R^{i} \Phi_{0}\right\rangle\right)
\end{aligned}
$$

and

$$
\begin{aligned}
& M_{x y}^{i \leftarrow f}\left(-\omega_{i, x}-i \gamma, \omega_{e, y}+i \gamma\right) \\
& =-\sum_{\rho \nu}\left(\left\langle\Phi_{0} L^{i} e^{-T}\left|\mu^{x}\right| e^{T} \Phi_{\rho}\right\rangle \sum_{n \geq 0} \frac{\left\langle\Phi_{\rho} \mid R^{n} \Phi_{0}\right\rangle\left\langle\Phi_{0} L^{n} \mid \Phi_{\nu}\right\rangle}{\Omega_{n i}-\omega_{i, x}-i \gamma}\left\langle\Phi_{\nu} e^{-T}\left|\mu^{x}\right| e^{T} R^{f} \Phi_{0}\right\rangle\right. \\
& \left.\quad+\left\langle\Phi_{0} L^{i} e^{-T}\left|\mu^{x}\right| e^{T} \Phi_{\rho}\right\rangle \sum_{n \geq 0} \frac{\left\langle\Phi_{\rho} \mid R^{n} \Phi_{0}\right\rangle\left\langle\Phi_{0} L^{n} \mid \Phi_{\nu}\right\rangle}{\Omega_{n i}+\omega_{e, y}+i \gamma}\left\langle\Phi_{\nu} e^{-T}\left|\mu^{x}\right| e^{T} R^{f} \Phi_{0}\right\rangle\right) .
\end{aligned}
$$

Here, $\rho$ spans the full excitation manifold; however, only the reference, singly, and doubly excited determinants survive for EOM-CCSD. Using the response intermediates defined $\operatorname{as}^{27}$

$$
\left[D^{x}\right]_{\nu}^{n}=\left\langle\Phi_{\nu} e^{-T}\left|\mu^{x}\right| e^{T} R^{n} \Phi_{0}\right\rangle
$$

and

$$
\left[\tilde{D}^{x}\right]_{\rho}^{n}=\left\langle\Phi_{0} L^{n} e^{-T}\left|\mu^{x}\right| e^{T} \Phi_{\rho}\right\rangle
$$


along with solutions, $X$ and $\tilde{X}$, of response equations given by

$$
\left(\bar{H}-E_{0}-(\omega+i \gamma)\right)_{\rho \nu}\left(X_{r e}+i X_{i m}\right)_{\nu}=D_{\rho}
$$

and

$$
\left(\tilde{X}_{r e}+i \tilde{X}_{i m}\right)_{\rho}\left(\bar{H}-E_{0}-(\omega+i \gamma)\right)_{\rho \nu}=\tilde{D}_{\nu}
$$

Eqs. (12) and (13) simplify to

$$
\begin{aligned}
& M_{x y}^{f \leftarrow i}\left(\omega_{i, x}+i \gamma,-\omega_{e, y}-i \gamma\right) \\
& \quad=-\sum_{\rho}\left(\left[\tilde{D}^{y}\right]_{\rho}^{f}\left[X^{x}\left(\omega_{i, x}+i \gamma\right)\right]_{\rho}^{i}+\left[\tilde{D}^{x}\right]_{\rho}^{f}\left[X^{y}\left(-\omega_{e, y}-i \gamma\right)\right]_{\rho}^{i}\right) \\
& \quad=-\sum_{\rho}\left(\left[\tilde{X}^{y}\left(-\omega_{e, y}-i \gamma\right)\right]_{\rho}^{f}\left[D^{x}\right]_{\rho}^{i}+\left[\tilde{X}^{x}\left(\omega_{i, x}+i \gamma\right)\right]_{\rho}^{f}\left[D^{y}\right]_{\rho}^{i}\right)
\end{aligned}
$$

and

$$
\begin{aligned}
& M_{x y}^{i \leftarrow f}\left(-\omega_{i, x}-i \gamma, \omega_{e, y}+i \gamma\right) \\
& \quad=-\sum_{\rho}\left(\left[\tilde{D}^{x}\right]_{\rho}^{i}\left[X^{y}\left(\omega_{e, y}+i \gamma\right)\right]_{\rho}^{f}+\left[\tilde{D}^{y}\right]_{\rho}^{i}\left[X^{x}\left(-\omega_{i, x}-i \gamma\right)\right]_{\rho}^{f}\right) \\
& \quad=-\sum_{\rho}\left(\left[\tilde{X}^{x}\left(-\omega_{i, x}-i \gamma\right)\right]_{\rho}^{i}\left[D^{y}\right]_{\rho}^{f}+\left[\tilde{X}^{y}\left(\omega_{e, y}+i \gamma\right)\right]_{\rho}^{i}\left[D^{x}\right]_{\rho}^{f}\right),
\end{aligned}
$$

where $X=X_{r e}+i X_{i m}$ and $\tilde{X}=\tilde{X}_{r e}+i \tilde{X}_{i m}$.

We solve damped response equations by modifying our standard DIIS procedure ${ }^{57,58}$ as described in the Supplementary Materials. Here, these damped response equations, Eqs. (16) and (17), implicitly employ the following resolvent equation:

$$
\sum_{n \geq 0} \frac{\left\langle\Phi_{\rho} \mid R^{n} \Phi_{0}\right\rangle\left\langle\Phi_{0} L^{n} \mid \Phi_{\nu}\right\rangle}{\Omega_{n i}-\omega-i \gamma}=\left\langle\Phi_{\rho}\left|\left(\bar{H}-E_{0}-\omega-i \gamma\right)^{-1}\right| \Phi_{\nu}\right\rangle .
$$

As a two-photon process, RIXS involves a virtual state (Fig. 1). Being a resonant process, the RIXS' virtual state is a real core-excited high-lying state, which is a metastable state (specifically, Feshbach resonance) with respect to electron ejection and is embedded in the valence ionization continuum. This presence of the continuum has a negative impact on the convergence of the response solutions, $X$ and $\tilde{X}$, in Eqs. (16) and (17). Note that the damped response theory framework only enables convergence of response equations for resonant photons but does not address the impact of the continuum. To circumvent this problem, we employ CVS scheme within the response manifold. 


\section{RIXS within fc-CVS-EOM-EE-CCSD damped response theory}

The fc-CVS-EOM-CCSD framework first imposes the frozen-core (fc) approximation to create distinct core occupied $(C)$, valence occupied $(O)$, and unoccupied $(V)$ subspaces. As reported in Ref. 14, the reference, $C V, C O V V$, and $C C V V$ excitation manifolds are then used for computing the fc-CVS-EOM-CCSD states. Note that in the fc-CVS-EOM-CCSD scheme, the $O V$ and $O O V V$ subspaces and thus the valence continuum have been projected out, which blocks the decay channels and makes the fc-CVS-EOM-CCSD states bound. This separation of the bound and continuum configurations within CVS is akin to diabatization.

In the context of calculating RIXS moments, we employ the CVS scheme to approximate the sum-over-state expressions in Eqs. (10) and (11) such that only the fc-CVS-EOM-EECCSD states are included in the sum. The fc-CVS-EOM-CCSD resolvent equation is given by

$$
\frac{\left\langle\Phi_{\rho} \mid \Phi_{0}\right\rangle\left\langle\Phi_{0} \mid \Phi_{\nu}\right\rangle}{-\omega-i \gamma}+\sum_{n}^{C V S} \frac{\left\langle\Phi_{\rho} \mid R^{n} \Phi_{0}\right\rangle\left\langle\Phi_{0} L^{n} \mid \Phi_{\nu}\right\rangle}{\Omega_{n i}-\omega-i \gamma}=\left\langle\Phi_{\rho}\left|\left(\bar{H}^{c v s}-E_{0}-\omega-i \gamma\right)^{-1}\right| \Phi_{\nu}\right\rangle,
$$

where the $\bar{H}^{c v s}$ is written in the basis of the reference, $C V, C O V V$, and $C C V V$ excitation manifolds. Eq. (21) reduces to

$$
\sum_{n}^{C V S} \frac{\left\langle\Phi_{\rho} \mid R^{n} \Phi_{0}\right\rangle\left\langle\Phi_{0} L^{n} \mid \Phi_{\nu}\right\rangle}{\Omega_{n i}-\omega-i \gamma}=\left\langle\Phi_{\rho}\left|\left(\bar{H}^{c v s}-E_{0}-\omega-i \gamma\right)^{-1}\right| \Phi_{\nu}\right\rangle-\frac{\delta_{0 \rho} \delta_{0 \nu}}{-\omega-i \gamma}
$$

The fc-CVS-EOM-EE-CCSD response equations are thus given by

$$
\left(\bar{H}-E_{0}-(\omega+i \gamma)\right)_{\rho \nu}\left(X_{r e}+i X_{i m}\right)_{\nu}=\left(1-\left|\Phi_{0}\right\rangle\left\langle\Phi_{0}\right|\right)_{\rho \lambda} D_{\lambda}
$$

and

$$
\left(\tilde{X}_{r e}+i \tilde{X}_{i m}\right)_{\rho}\left(\bar{H}-E_{0}-(\omega+i \gamma)\right)_{\rho \nu}=\tilde{D}_{\lambda}\left(1-\left|\Phi_{0}\right\rangle\left\langle\Phi_{0}\right|\right)_{\lambda \nu},
$$

where $\rho, \lambda$, and $\nu$ span the reference, $C V, C O V V$, and $C C V V$ excitation manifolds.

\section{COMPUTATIONAL DETAILS}

The validation calculations for $\mathrm{LiH}$ were carried out using the STO-3G basis and $r_{L i H}=1.6 \AA$. Water calculations were carried out using the same setup as in Ref. 16. Specifically, we computed RIXS cross sections including the lowest 20 excited states, using 
the geometry $r_{O H}=0.95421 \AA, \angle_{H O H}=104.78^{\circ}$ ) and the basis set from Ref. 16 (also given in the Supplementary Material). We use the EOM-EE-CCSD results reported at the incident photon frequency of $535.74 \mathrm{eV}$ that is resonant with $1 s \rightarrow 4 a_{1}$ core excitation in water. The corresponding fc-CVS-EOM-EE-CCSD incident photon frequency used in our calculations is $535.23 \mathrm{eV}$.

The calculations for neutral benzene were carried out at the geometry optimized with RIMP2/cc-pVTZ. The calculations for the cation were carried out at two different geometries: one of the neutral (referred to as Franck-Condon (FC) structure) and one of the cation (optimized with EOM-IP-CCSD/cc-pVTZ). We considered the optimized geometry of the ${ }^{2} \mathrm{~B}_{3 g}$ state, which is the lowest Jahn-Teller state (this structure can be described as "elongated", in terms of Ref. 59 or "acute $D_{2 h}$ ", in terms of Ref. 60. The optimized structure of the second Jahn-Teller state, ${ }^{2} \mathrm{~B}_{2 g}$, is nearly degenerate with the ${ }^{2} \mathrm{~B}_{3 g}$ structure and the barrier separating the two minima is well below zero-point energy ${ }^{60}$. Consequently, benzene cation structure can be described as free pseudo-rotation ${ }^{60}$. We used the $6-311(2+,+) \mathrm{G}^{* *}$ basis with the uncontracted carbon core (denoted as 6-311(2+,+) $\mathrm{G}^{* *}(\mathrm{uC})$ ).

Depending on molecular orientation, symmetry labels corresponding to the same orbital or vibrational mode may be different. Q-Chem's standard molecular orientation is different from that of Mulliken ${ }^{61}$. For example, Q-Chem places water molecule in the $x z$ plane instead of the $y z$. Consequently, for $\mathrm{C}_{2 v}$ symmetry, $b_{1}$ and $b_{2}$ labels are flipped. Q-Chem places benzene in the $x y$ plane (with the x-axis passing through the carbon atoms) instead of the $y z$ plane. Consequently, the conventional $b_{1 u}$ and $b_{1 u}$ labels are flipped in Q-Chem. More details can be found at http://iopenshell.usc.edu/resources/howto/symmetry/

The calculations of XAS were carried out using fc-CVS-EOM-EE-CCSD. For the cation, we used restricted and unrestricted open-shell Hartree-Fock (ROHF and UHF, respectively) references and the difference between the two calculations was found to be small. Below we report the ROHF results. The XES transitions between different core and valence states were computed as transitions between valence and core-ionized states of the neutral reference, as suggested in Ref. 53. The valence states were described by EOM-IP-CCSD with frozen core and the core states were described by fc-CVS-EOM-IP-CCSD. The orbital character of the XAS and XES transitions was analyzed using Natural Transition Orbitals computed with the libwfa module ${ }^{62}$ and Dyson orbitals ${ }^{63,64}$. 
The calculations of RIXS were done with the UHF, using the new fc-CVS-EOM-EECCSD scheme. RIXS calculations were performed with $\epsilon=0.005 \mathrm{au}=0.136 \mathrm{eV}$ for peak A of the neutral and for the valence pump of the cation and with $\epsilon=0.01 \mathrm{a} . \mathrm{u}$. $=0.272 \mathrm{eV}$ for peak $\mathrm{B}$ of the neutral.

The stick spectra were convoluted with normalized Gaussian functions with FWHM = $0.25 \mathrm{eV}$ :

$$
\begin{array}{r}
g(x)=\frac{1}{\sigma \sqrt{2 \pi}} \exp ^{-0.5 *\left(x-x_{0}\right)^{2} / \sigma^{2}} \\
F W H M=2 \sqrt{2 \ln (2))} \sigma \approx 2.35482 \sigma
\end{array}
$$

\section{RESULTS AND DISCUSSION}

\section{A. Validation and benchmarks}

The calculation of RIXS cross sections using damped response theory combined with CVS is implemented in the development version of the Q-Chem electronic structure package ${ }^{54,55}$. Our production-level implementation builds upon the fc-CVS-EOM-CCSD suite of $\operatorname{codes}^{14}$ and the non-linear response module ${ }^{27}$. The programmable equations are implemented using libtensor $^{65}$. The implementation allows calculation of RIXS cross sections for both closedand open-shell references. We validated our analytic damped response-theory approach against numerical sum-over-state calculations for the first excited state of $\mathrm{LiH}$ using the STO-3G basis; the results are given in the Supplementary Materials.

To quantify the effect of using CVS within the response equations on the RIXS spectra, we compare the fc-CVS-EOM-EE-CCSD and standard EOM-EE-CCSD results for the water molecule. The incident photon frequency in each calculation is resonant with the $1 s_{O} \rightarrow 4 a_{1}$ core excitation. For this incident photon, the dominant RIXS transitions correspond to final states $1 B_{2}, 2 A_{1}$, and $3 A_{1}$ with orbital characters of $1 b_{2} \rightarrow 4 a_{1}, 3 a_{1} \rightarrow 4 a_{1}$, and $1 b_{2} \rightarrow 2 b_{2}$ (in Q-Chem's symmetry notation), respectively, as presented in Table I. Table I reveals that using the fc-CVS scheme has a minor impact on the RIXS cross sections for these dominant transitions in the spectrum. However, we note that the effect on the cross sections of several minor bands is larger. Importantly, the appearance of the overall computed spectrum is not affected, as illustrated in Fig. 3. 
TABLE I: Comparison of EOM-EE-CCSD and fc-CVS-EOM-EE-CCSD excitation energies and RIXS cross sections for $\mathrm{H}_{2} \mathrm{O}(\gamma=0.0045563$ a.u. $)$. Incident photon frequency for EOM-EE-CCSD calculations $=19.6881298$ a.u. $(535.74 \mathrm{eV})$. Incident photon frequency for fc-CVS-EOM-EE-CCSD calculations $=19.6692301$ a.u $(535.23 \mathrm{eV})$. The last column gives the ratio of computed RIXS cross sections with the two methods.

\begin{tabular}{c|cc|ccc}
\hline \hline Ex. State $^{a}$ & \multicolumn{4}{|c|}{ EOM-EE-CCSD } & \multicolumn{3}{c}{ fc-CVS-EOM-EE-CCSD } \\
\hline & Ex. E. & $\sigma^{R I X S}\left(0^{\circ}\right)$ & Ex. E. & $\sigma^{R I X S}\left(0^{\circ}\right)$ & ratio \\
& $\mathrm{eV}$ & a.u. & $\mathrm{eV}$ & a.u. & \\
\hline $1 \mathrm{~B}_{2}$ & 7.41 & 0.023556 & 7.40 & 0.023646 & 1.00 \\
$1 \mathrm{~A}_{2}$ & 9.16 & 0.000491 & 9.15 & 0.000180 & 0.37 \\
$2 \mathrm{~A}_{1}$ & 9.74 & 0.007563 & 9.74 & 0.007645 & 1.01 \\
$2 \mathrm{~B}_{2}$ & 10.01 & 0.000759 & 10.01 & 0.000053 & 0.07 \\
$3 \mathrm{~A}_{1}$ & 10.10 & 0.001288 & 10.10 & 0.001314 & 1.02 \\
$3 \mathrm{~B}_{2}$ & 10.39 & 0.000253 & 10.38 & 0.000257 & 1.02 \\
$2 \mathrm{~A}_{2}$ & 10.80 & 0.000015 & 10.79 & 0.000029 & 1.93 \\
$4 \mathrm{~B}_{2}$ & 11.21 & 0.000159 & 11.21 & 0.000009 & 0.05 \\
$4 \mathrm{~A}_{1}$ & 11.22 & 0.000011 & 11.21 & 0.000011 & 1.04 \\
$5 \mathrm{~B}_{2}$ & 11.30 & 0.000062 & 11.30 & 0.000060 & 0.97 \\
$3 \mathrm{~A}_{2}$ & 11.43 & 0.000038 & 11.42 & 0.000010 & 0.25 \\
$1 \mathrm{~B}_{1}$ & 11.50 & 0.000430 & 11.50 & 0.000130 & 0.30 \\
$6 \mathrm{~B}_{2}$ & 11.68 & 0.000092 & 11.67 & 0.000016 & 0.18 \\
$5 \mathrm{~A}_{1}$ & 11.69 & 0.000002 & 11.69 & 0.000002 & 1.10 \\
$7 \mathrm{~B}_{2}$ & 11.72 & 0.000076 & 11.71 & 0.000016 & 0.21 \\
$4 \mathrm{~A}_{2}$ & 11.83 & 0.000155 & 11.81 & 0.000005 & 0.03 \\
$8 \mathrm{~B}_{2}$ & 12.20 & 0.000641 & 12.20 & 0.000013 & 0.02 \\
$6 \mathrm{~A}_{1}$ & 12.26 & 0.000046 & 12.26 & 0.000045 & 0.98 \\
$7 \mathrm{~A}_{1}$ & 12.66 & 0.000113 & 12.66 & 0.000118 & 1.04 \\
$9 \mathrm{~B}_{2}$ & 13.07 & 0.000298 & 13.06 & 0.000191 & 0.64 \\
\hline & $\mathrm{Q}-\mathrm{Chem} \mathrm{s}$ symmetry notations. &
\end{tabular}

\section{B. Illustrative calculation: RIXS of benzene and benzene cation}

\section{Benzene}

Modeling X-ray spectra for benzene is challenging given its high symmetry, multiple core orbitals, and the need for highly diffuse functions for converged results. Figure 4 shows 


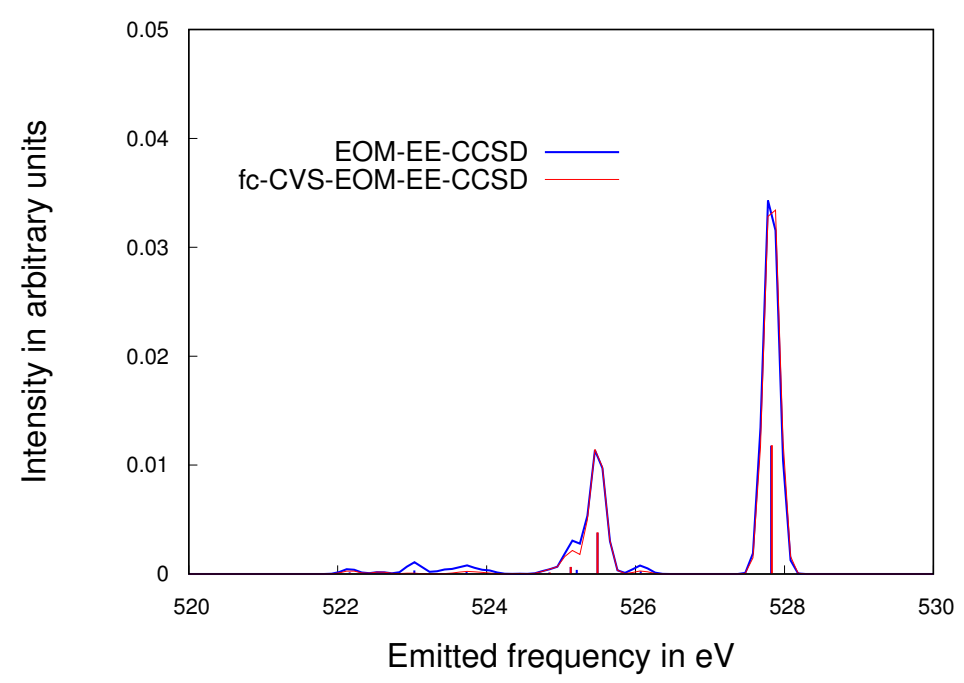

FIG. 3: Comparison of EOM-EE-CCSD and fc-CVS-EOM-EE-CCSD RIXS spectra for the incident photon resonant with the $1 s \rightarrow 4 a_{1}$ core excitation in water. $\left(\mathrm{FWHM}=0.33 \mathrm{eV} ; \sigma=\frac{5}{\sqrt{2}}\right)$

occupied molecular orbital diagram of benzene; the respective vertical ionization energies (IEs) computed at the geometry of the neutral are collected in Table S2 of the Supplementary Materials. Due to symmetry, all six $1 \mathrm{~s}_{C}$ core orbitals are delocalized, however, the splittings of the respective IEs are small (all six lie within $0.08 \mathrm{eV}$ ) because of their compactness. Table S2 also lists the IEs of the valence orbitals; the lowest IE of $9.2 \mathrm{eV}$ corresponds to the degenerate pair of $\pi$ orbitals, which give rise to the Jahn-Teller distortion. These values of IEs highlight the maximum range of energy loss expected in XES and RIXS calculations. 


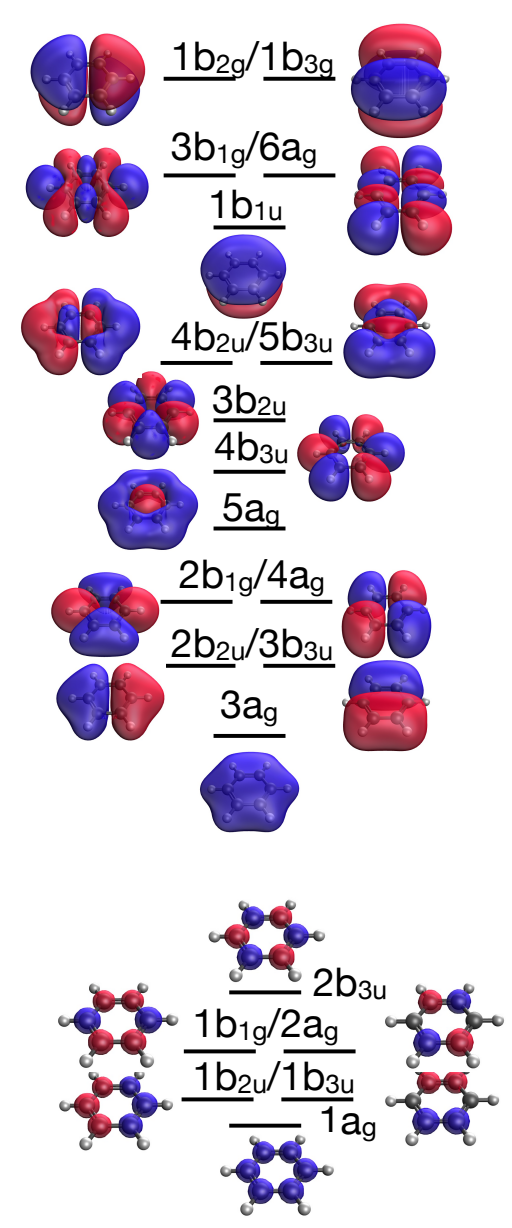

FIG. 4: Occupied molecular orbitals diagram for benzene.

The XAS spectrum of neutral benzene is shown in Fig. 5; the respective transition energies and strengths are collected in Table S3 of the Supplementary Materials. The positions and relative intensities of the main peaks agree well with the experiment ${ }^{66,67}$ after a systematic shift of $-0.8 \mathrm{eV}$ is applied. Fig. 5 also shows the leading NTOs for the first two peaks; the NTOs for other transitions are collected in Supplementary Materials. The NTOs reveal that peak $\mathrm{A}$ is derived from the $1 s_{C} \rightarrow \pi^{*} B_{1 u}$ transition, whereas doubly degenerate peak B corresponds to the $1 s_{C} \rightarrow R y B_{2 u} / B_{3 u}$ transition. The NTO analysis shows that excitation of peak A creates a hole in the $2 a_{g}$ and $1 b_{1 g}$ core orbitals, whereas the excitation of peak B creates holes in $\mathrm{b}_{1 u} / \mathrm{b}_{2 u}$ and on $2 a_{g}$. The weights of the NTOs (i.e., respective $\sigma^{2}$ values) quantify the relative contribution of each hole. Although the energies of the core orbitals giving rise to peak $\mathrm{A}$ and peak $\mathrm{B}$ are very close, their different symmetries have profound effect on the shape of the RIXS energy loss spectra corresponding to pumping these two 


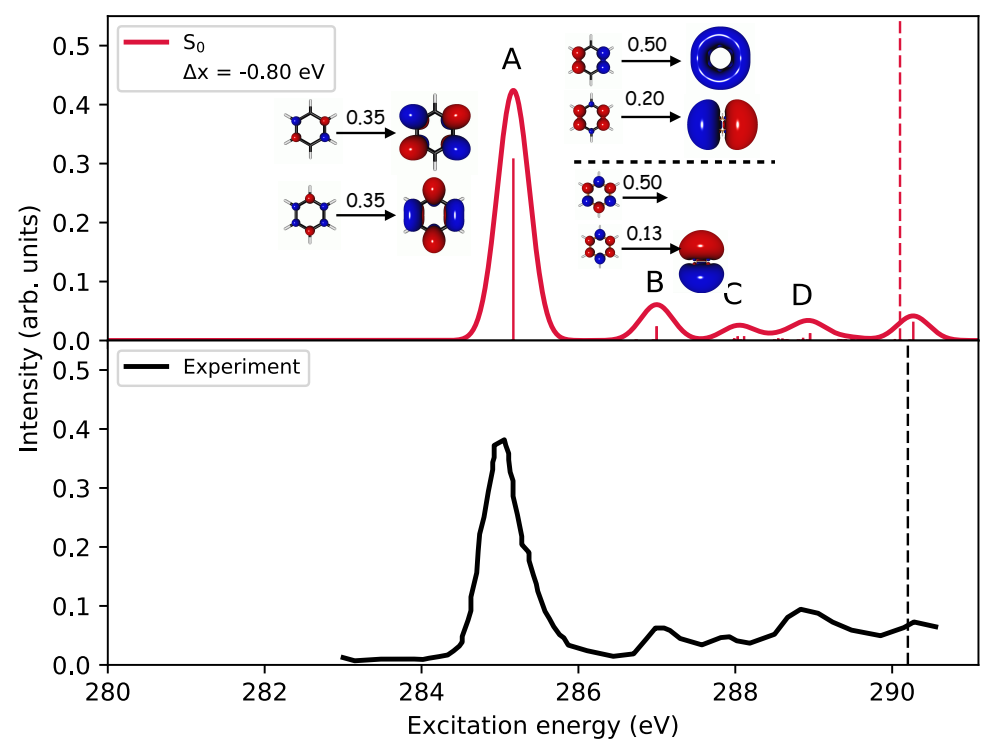

FIG. 5: XAS spectrum of benzene computed with fc-CVS-EOM-CCSD/6-311 $(2+,+) \mathrm{G}^{* *}(\mathrm{uC})$ obtained by convolution of the spectral data in Table S3 with a Gaussian function (FWHM $=0.8$ $\mathrm{eV}$ ). Dashed vertical lines correspond to the IEs. The energy shift required to align the NEXAFS profiles with the experimental one is $0.8 \mathrm{eV}$. The computed IE has been shifted by the same amount as used to align the NEXAFS profiles. NTOs and their weights are shown for peak A and peak B. The experimental spectrum is from Ref. 66 .

peaks.

As suggested by Fig. 2, the RIXS process can be crudely described as a union of XAS excitation followed by the emission via XES-like transitions. Assuming that the excited electron does not strongly perturb the $N$-1-electron core, the energy loss spectra in RIXS of the neutral benzene can be approximated by computing XES transitions between the core and valence ionized states. Depending on the character of the pumping transition, the ensuing XES transitions will involve different core orbitals. The NTO analysis of of the XAS spectra on benzene shown in Fig. 5 and in Supplementary Materials show which core orbitals give rise to peaks A and B. We use these orbitals (and the respective NTO weights) to compute the corresponding XES transitions; the resulting energy loss spectra are shown in Fig. 6. The two XES spectra show significant differences: the relaxation to the hole of peak A yields only two features, whereas the relaxation to the hole of the peak B shows more features dominated by the one due to the relaxation of the HOMO to the core hole around $282 \mathrm{eV}$. This is not surprising as the symmetry of the core hole dictates the symmetry of the 
valence orbitals which are active in XES (or RIXS) transitions. For example, the presence of the $\mathrm{b}_{3 u}$ core hole will allow only the relaxation from $a_{g}, b_{1 g}$, and $\mathrm{b}_{2 g}$ valence levels (in $D_{2 h}$ group, only $\mathrm{B}_{1 u}, \mathrm{~B}_{2 u}$, and $\mathrm{B}_{3 u}$ transitions are optically allowed). These calculations suggest that the RIXS energy-loss spectra will also show strong dependence on the pumping frequency.

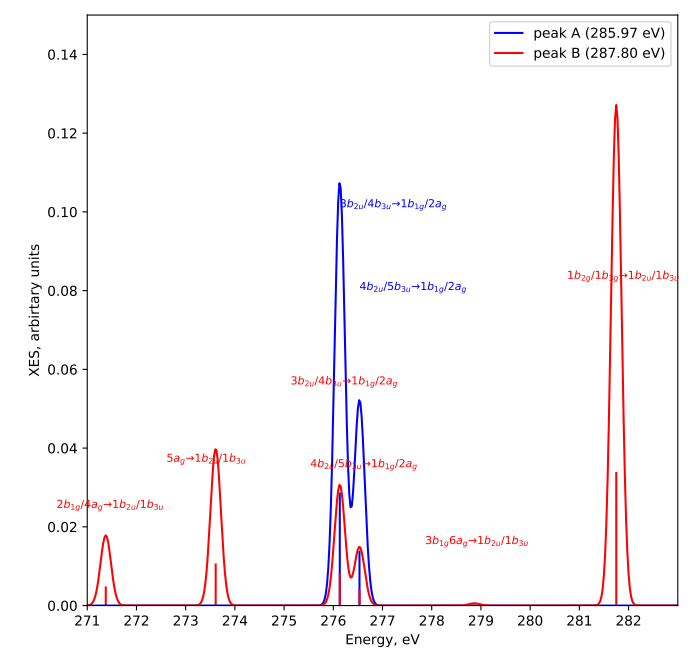

FIG. 6: XES transitions in the neutral benzene corresponding to pumping peak A (blue) and peak B (red) computed with fc-CVS-EOM-IP-CCSD and fc-EOM-IP-CCSD and 6-311 $(2+,+) \mathrm{G}^{* *}(\mathrm{uC})$.

The computed RIXS energy loss spectra corresponding to pumping peak A and peak B computed with fc-CVS-EOM-EE-CCSD are shown in Fig. 7. As one can see, the differences in RIXS spectra corresponding to the resonant $1 \mathrm{~s}_{C} \rightarrow \pi^{*}$ core excitation (peak A) and $1 s_{C} \rightarrow R y$ core excitation (peak B) are indeed significant. Note that the two-photon RIXS process for $D_{2 h}$ benzene dictates that only gerade transitions are allowed. Fig. 7 also highlights the differences of the real RIXS spectra with the two energy loss spectra computed as XES transitions corresponding to core holes of the XAS peaks A and B. As one can see, the dominant features of the RIXS spectra are predicted reasonably well by the estimates based on the XES transitions, however, quantitatively, proper RIXS spectra have more features; the positions and relative intensities of the dominant peaks are also shifted. These differences highlight the effect of the electron excited by the pump on the core relaxation 
process.

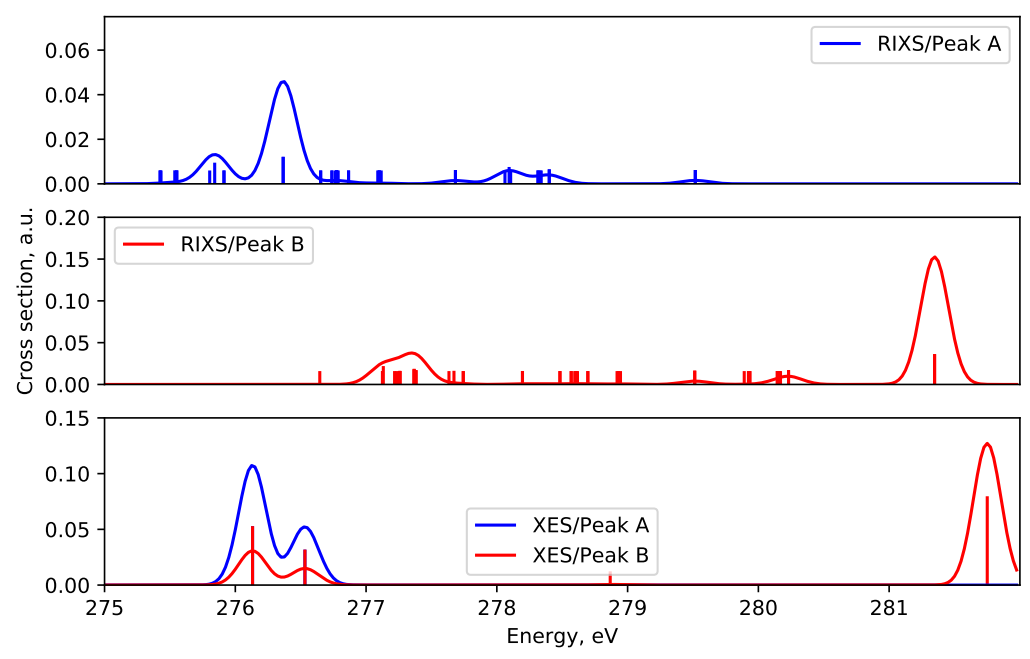

FIG. 7: Benzene RIXS spectra corresponding to pumping peak A (285.97 eV) and peak B (287.80 $\mathrm{eV}$ ) with $\theta=0$ and the corresponding XES spectra (see text).

To further analyze RIXS spectra, below we discuss which excited states of the neutral contribute to the RIXS features. The RIXS spectrum for incident photon resonant with XAS peak A $\left(\omega_{i}=285.97 \mathrm{eV}\right)$ shows three dominant peaks centered at $7.9 \mathrm{eV}, 9.6 \mathrm{eV}$, and $10.1 \mathrm{eV}$ energy losses. Whereas the $7.9 \mathrm{eV}$ and $10.1 \mathrm{eV}$ energy losses correspond to the transitions to valence $3 \mathrm{~A}_{g}$ and $8 \mathrm{~A}_{g}$ states, the dominant peak at $9.6 \mathrm{eV}$ is due to doubly degenerate transition to $10 B_{2 g}$ and $10 B_{3 g}$ states.

In contrast to peak A pump, the RIXS spectrum for incident photon resonant with peak B $\left(\omega_{i}=287.80 \mathrm{eV}\right)$ shows six leading transitions corresponding to $6.45 \mathrm{eV}$ (doubly degenerate transition to $1 \mathrm{~B}_{2 g}$ and $1 \mathrm{~B}_{3 g}$ ), $7.56 \mathrm{eV}$ (doubly degenerate transition to $2 \mathrm{~B}_{2 g}$ and $2 \mathrm{~B}_{3 g}$ ), 10.42 $\mathrm{eV}$ (doubly degenerate transition to $9 \mathrm{~A}_{g}$ and $8 \mathrm{~B}_{1 g}$ ), $10.43 \mathrm{eV}$ (doubly degenerate transition to $11 \mathrm{~B}_{2 g}$ and $11 \mathrm{~B}_{3 g}$ ), $10.43 \mathrm{eV}$ (transition to $9 \mathrm{~B}_{1 g}$ ), and $10.67 \mathrm{eV}$ (transition to $12 \mathrm{~B}_{3 g}$ ) energy losses. The simplified RIXS orbital picture would be a sum of two $b_{2 u} / b_{3 u}$ transitions as the $1 s_{C} \rightarrow R y$ core excitation has $b_{2 u} / b_{3 u}$ symmetry. 


\section{Benzene cation}

XAS and RIXS spectra of open-shell species derived by the ionization of closed-shell molecules acquire new, distinguishing features due to the presence of the valence hole. The excitation of the core electrons can populate this valence hole, giving rise to a characteristic lower energy peak in the XAS spectra of the open-shell species. The resonant excitation of this peak in RIXS provides a means to get the energy-loss spectrum characteristic of the open-shell species. In particular, the characteristic features of this RIXS energy-loss spectrum for the open-shell species and the XES spectrum of the parent closed-shell species with the same core hole are expected to be similar when computed at the same geometry. Here, we illustrate these essential relationships between the closed-shell molecules and the respective ionized species by considering XAS, XES, and RIXS of benzene and its cation.

Benzene cation is a classic Jahn-Teller system ${ }^{60}$ with doubly degenerate (at the FranckCondon) geometry ground state. Jahn-Teller distortions (of $0.2 \mathrm{eV}$ ) lead to two nearly degenerate minima, with the barrier between them well below zero point energy ${ }^{60}$.

Fig. 8 shows XAS spectra of the cation (computed for ${ }^{2} \mathrm{~B}_{3 g}$ state) and the respective NTOs for the lowest spectral feature. At the geometry of the neutral, Peak A corresponds to the excitation to the valence hole (transitions between $1 b_{2 u} / 1 b_{3 u}$ core and $1 b_{3 g} / 1 b_{2 g}$ HOMO), while peaks $\mathrm{C}$ and $\mathrm{D}$ resemble the transitions in the neutral species (i.e., peaks $\mathrm{A}$ and $\mathrm{B}$ of the XAS of the neutral). Geometric relaxation has a rather small effect on the spectral shape. Peak A is blue-shifted by $0.3 \mathrm{eV}$. The results for all spectral lines are given in Supplementary Materials.

Fig. 9 compares the emitted frequency spectra computed as XES transitions to the $1 b_{2 u} / 1 b_{3 u}$ core hole and the RIXS spectrum of benzene cation computed for pumping transition corresponding to the excitation into the valence hole (i.e., with incident photon resonant with peak A of the cation). The RIXS spectra were computed using 6 excited states in each gerade irrrep. As expected, the two dominant features are similar - the elastic peak and the transition to the $2 \mathrm{~A}_{g}$ state are slightly blue shifted by about $0.3 \mathrm{eV}$. Geometric relaxation has relatively small effect on the position and intensities of these two major features. 


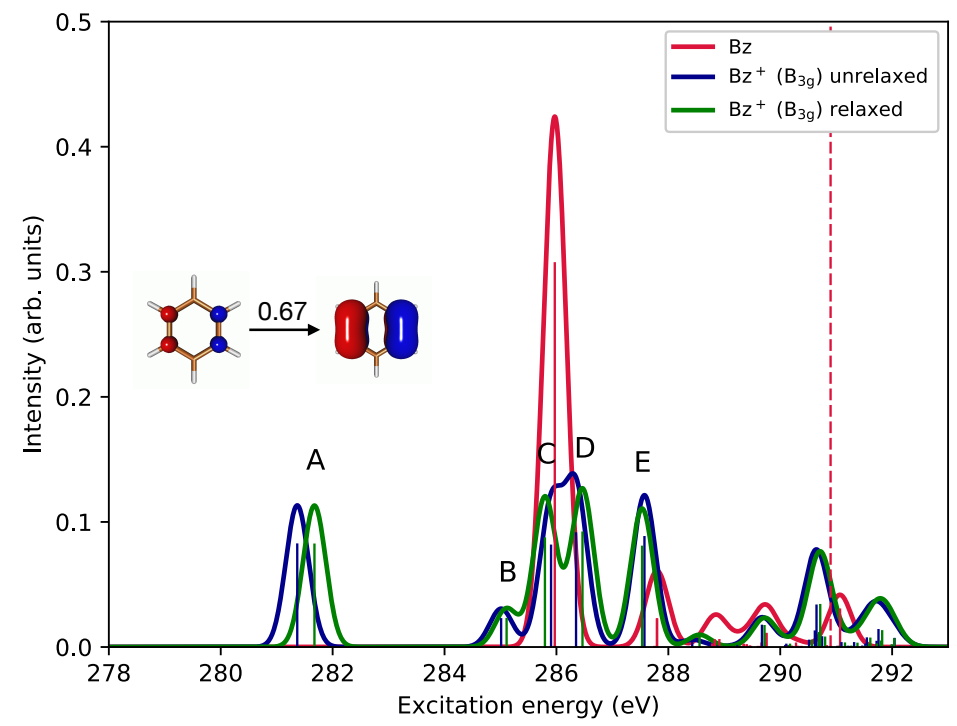

FIG. 8: XAS spectra of neutral benzene and its cation computed with fc-CVS-EOMEE-CCSD/6$311(2+,+) \mathrm{G}^{* *}(\mathrm{uC})$ at the Franck-Condon geometry and at the optimized geometry of the ${ }^{2} \mathrm{~B}_{3 g}$ ionized state. A Gaussian convolution function $(\mathrm{FWHM}=0.8 \mathrm{eV})$ was used.
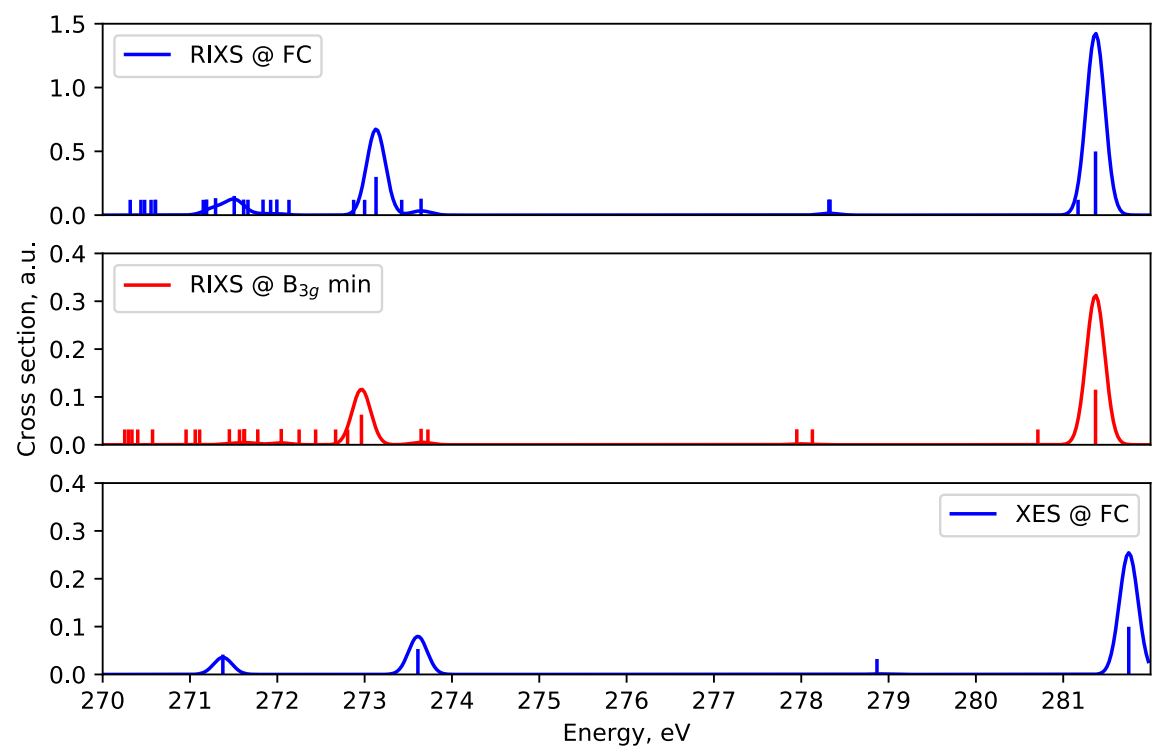

FIG. 9: Benzene cation $\left({ }^{2} \mathrm{~B}_{3 g}\right)$. RIXS spectra corresponding to the valence pump $(281.37 \mathrm{eV})$ computed at the FC geometry and at the optimized geometry of the cation $\left({ }^{2} \mathrm{~B}_{3 g}\right.$ minimum) at $\theta=0$ and the corresponding XES spectra. 


\section{CONCLUSIONS}

In this contribution we present an extension of dumped response theory for calculating RIXS cross sections using CVS scheme within the response equations. Our primary aim was to address problematic divergence and erratic behavior of response solutions in the X-ray frequency range, which is needed for RIXS calculations. The cause for this problematic behavior is the embedding of high-lying virtual states in the valence-ionization continuum. We have extended a newly developed fc-CVS-EOM-EE-CCSD flavor of EOM-EE theory to RIXS calculations by combining the damped response theory and CVS truncation of the response space. This method exploits the ability of CVS to decouple the valence-excitation and core-excitation configurations of the excitation manifold, thereby blocking the autoionization decay channels for the virtual (i.e., response) states. The numeric results demonstrate that this approach solves the problematic divergence of the response solutions, while not affecting the quality of the computed RIXS spectrum. Our fc-CVS-EOM-EE-CCSD implementation can treat both closed- and open-shell species, which we exemplified by calculating the RIXS energy-loss spectra of (closed-shell) benzene and its (open-shell) cation. We analyzed the XAS, XES, and RIXS spectra of these species in terms of molecular orbitals and demonstrated the qualitative differences and similarities between their spectra. 


\section{Supplementary Material}

This document contains (1) programmable equations for iterative solutions of damped response equations, (2) $\mathrm{LiH}$ data for validation of code with numerical sum-over-state RIXS moments, (3) relevant Cartesian coordinates, (4) raw data for XAS, XES, and RIXS spectra including relevant NTOs, and (5) basis set used for water.

\section{Acknowledgments}

This work was supported by the U.S. National Science Foundation (No. CHE-1856342). A.I.K. is a grateful recipient of the Simons Fellowship in Theoretical Physics and Mildred Dresselhaus Award from CFEL/DESY, which supported her sabbatical stay in Germany. M.L.V., R.F., and S.C. acknowledge financial support from DTU Chemistry and from the Independent Research Fund Denmark - DFF-Forskningsprojekt2 grant no. 7014-00258B. S.C. also acknowledges the European Union's Horizon 2020 Research and Innovation Programme under the Marie Sklodowska-Curie Grant Agreement No. 765739, "COSINE European Training Network on COmputational Spectroscopy In Natural sciences and Engineering."

1 S.M. Butorin, J. Electron. Spectrosc. Relat. Phenom. 110-111, 213 (2000).

2 F. de Groot, Chem. Rev. 101, 1779 (2001).

3 A. Kotani and S. Shin, Rev. Mod. Phys. 73, 203 (2001).

4 L.J.P. Ament, M. van Veenendaal, T. P. Devereaux, J.P. Hill, and J. van den Brink, Rev. Mod. Phys. 83, 705 (2011).

5 T. Schmitt, F.M.F. de Groot, and J. Rubensson, J. Synchrotron Rad. 21, 1065 (2014).

6 S. Mobilio, F. Boscherini, and C. Meneghini, editors, Synchrotron Radiation: Basics, Methods and Applications. Springer, 2014.

7 J.A. van Bokhoven and C. Lamberti, editors, X-Ray Absorption and X-ray Emission Spectroscopy; Theory and Applications. Wiley \& Sons, 2016.

8 U. Bergmann, V.K. Yachandra, and J. Yano, editors, X-Ray Free Electron Lasers: Applications in Materials, Chemistry and Biology, Number 18 in Energy and Environment Series. Royal 
Society of Chemistry, 2017.

9 M. Nisoli, P. Decleva, F. Calegari, A. Palacios, and F. Martín, Chem. Rev. 117, 10760 (2017).

10 P. Norman and A. Dreuw, Chem. Rev. 118, 7208 (2018).

11 T. Fransson, Y. Harada, N. Kosugi, N.A. Besley, B. Winter, J.J. Rehr, L.G.M. Pettersson, and A. Nilsson, Chem. Rev. 116, 7551 (2016).

12 O. Kostko, B. Bandyopadhyay, and M. Ahmed, Annu. Rev. Phys. Chem. 67, 19 (2016).

13 A. Sadybekov and A. I. Krylov, J. Chem. Phys. 147, 014107 (2017).

14 M. L. Vidal, X. Feng, E. Epifanovski, A. I. Krylov, and S. Coriani, J. Chem. Theory Comput. 15, 3117 (2019).

15 D.R. Rehn, A. Dreuw, and P. Norman, J. Chem. Theory Comput. 13, 5552 (2017).

16 R. Faber and S. Coriani, J. Chem. Theory Comput. 15, 520 (2019).

17 A.D. Buckingham and P. Fischer, Phys. Rev. A 61, 035801 (2000).

18 K. Kristensen, J. Kauczor, A. J. Thorvaldsen, P. Jørgensen, T. Kjaergaard, and A. Rizzo, J. Chem. Phys. 134, 214104 (2011).

19 K. Emrich, Nucl. Phys. A351, 379 (1981).

20 J. F. Stanton and R. J. Bartlett, J. Chem. Phys. 98, 7029 (1993).

21 A. I. Krylov, Annu. Rev. Phys. Chem. 59, 433 (2008).

22 R. J. Bartlett, Mol. Phys. 108, 2905 (2010).

23 K. Sneskov and O. Christiansen, WIREs: Comput. Mol. Sci. 2, 566 (2012).

24 R. J. Bartlett, WIREs: Comput. Mol. Sci. 2, 126 (2012).

25 L.V. Slipchenko, J. Phys. Chem. A 114, 8824 (2010).

26 T. Helgaker, S. Coriani, P. Jørgensen, K. Kristensen, J. Olsen, and K. Ruud, Chem. Rev. 112, $543(2012)$.

27 K. D. Nanda and A. I. Krylov, J. Chem. Phys. 142, 064118 (2015).

28 K. D. Nanda and A. I. Krylov, J. Chem. Phys. 146, 224103 (2017).

29 K. Nanda and A. I. Krylov, J. Phys. Chem. Lett. 8, 3256 (2017).

30 K. Nanda and A. I. Krylov, J. Chem. Phys. 149, 164109 (2018).

31 M. de Wergifosse, C. G. Elles, and A. I. Krylov, J. Chem. Phys. 146, 174102 (2017).

32 M. de Wergifosse, A. L. Houk, A. I. Krylov, and C. G. Elles, J. Chem. Phys. 146, 144305 (2017).

33 O. Christiansen, J. Gauss, and B. Schimmelpfennig, Phys. Chem. Chem. Phys. 2, 965 (2000). 
34 K. Klein and J. Gauss, J. Chem. Phys. 129, 194106 (2008).

35 E. Epifanovsky, K. Klein, S. Stopkowicz, J. Gauss, and A. I. Krylov, J. Chem. Phys. 143, $064102(2015)$.

36 P. Pokhilko, E. Epifanovsky, and A.I. Krylov, J. Chem. Phys. (2019), in press; preprint: https://doi.org/10.26434/chemrxiv.8064458.v1.

37 A. Tajti and P.G. Szalay, J. Chem. Phys. 131, 124104 (2009).

38 T. Ichino, J. Gauss, and J. F. Stanton, J. Chem. Phys. 130, 174105 (2009).

39 S. Faraji, S. Matsika, and A. I. Krylov, J. Chem. Phys. 148, 044103 (2018).

40 P. B. Rozyczko, S. A. Perera, M. Nooijen, and R. J. Bartlett, J. Chem. Phys. 107, 6736 (1997).

41 C. Hättig, O. Christiansen, S. Coriani, and P. Jørgensen, J. Chem. Phys. 109, 9237 (1998).

42 K. D. Nanda and A. I. Krylov, J. Chem. Phys. 145, 204116 (2016).

43 K. Nanda, A. I. Krylov, and J. Gauss, J. Chem. Phys. 149, 141101 (2018).

44 S. Coriani, O. Christiansen, T. Fransson, and P. Norman, Phys. Rev. A 85, 022507 (2012).

45 S. Coriani, T. Fransson, O. Christiansen, and P. Norman, J. Chem. Theory Comput. 8, 1616 (2012).

46 D. Zuev, E. Vecharynski, C. Yang, N. Orms, and A. I. Krylov, J. Comput. Chem. 36, 273 (2015).

47 B. Peng, P.J. Lestrange, J.J. Goings, M. Caricato, and X. Li, J. Chem. Theory Comput. 11, $4146(2015)$.

48 S. Coriani and H. Koch, J. Chem. Phys. 143, 181103 (2015).

49 S. Coriani and H. Koch, J. Chem. Phys. 145, 149901 (2016).

50 H. Sekino and R. J. Bartlett, Int. J. Quant. Chem. 26, 255 (1984).

51 H. Koch, H.J.Aa. Jensen, P. Jørgensen, and T. Helgaker, J. Chem. Phys. 93, 3345 (1990).

52 L.S. Cederbaum, W. Domcke, and J. Schirmer, Phys. Rev. A 22, 206 (1980).

53 R. Faber and S. Coriani, Phys. Chem. Chem. Phys. (2019), submitted.

54 A. I. Krylov and P. M. W. Gill, WIREs: Comput. Mol. Sci. 3, 317 (2013).

55 Shao, Y.; Gan, Z.; Epifanovsky, E.; Gilbert, A.T.B.; Wormit, M.; Kussmann, J.; Lange, A.W.; Behn, A.; Deng, J.; Feng, X., et al., Mol. Phys. 113, 184 (2015).

56 M.J. Paterson, O. Christiansen, F. Pawlowski, P. Jørgensen, C. Hättig, T. Helgaker, and P. Salek, J. Chem. Phys. 124, 054332 (2006).

57 P. Pulay, Chem. Phys. Lett. 73, 393 (1980). 
58 G.E. Scuseria, T.J. Lee, and H.F. Schaefer, Chem. Phys. Lett. 130, 236 (1986).

59 H. Tachikawa, J. Phys. Chem. A 122, 4121 (2018).

60 R. Lindner, K. Müller-Dethlefs, E. Wedum, K. Haber, and E.R. Grant, Science 271, 1698 (1996).

61 R.S. Mulliken, J. Chem. Phys. 23, 1997 (1955).

62 F. Plasser, M. Wormit, and A. Dreuw, J. Chem. Phys. 141, 024106 (2014).

63 C. M. Oana and A. I. Krylov, J. Chem. Phys. 127, 234106 (2007).

64 M. L. Vidal, A. I. Krylov, and S. Coriani, Phys. Chem. Chem. Phys. (2019), submitted.

65 E. Epifanovsky, M. Wormit, T. Kuś, A. Landau, D. Zuev, K. Khistyaev, P. U. Manohar, I. Kaliman, A. Dreuw, and A. I. Krylov, J. Comput. Chem. 34, 2293 (2013).

66 D. Menzel, G. Rocker, H.-P. Steinrück, D. Coulmanand P. A. Heimann, W. Huber, P. Zebisch, and D. R. Lloyd, J. Chem. Phys. 96, 1724 (1992).

67 E. E. Rennie, B. Kempgens, H. M. Köppe, U. Hergenhahn, J. Feldhaus, B. S. Itchkawitz, A. L. D. Kilcoyne, A. Kivimäki, K. Maier, M. N. Piancastelli, M. Polcik, A. Rüdel, and A. M. Bradshaw, J. Chem. Phys. 113, 7362 (2000). 